\title{
POLYNOMIALS DEFINED BY A DIFFERENCE SYSTEM
}

\author{
BY GLEN BAXTER ${ }^{1}$
}

Communicated by Paul C. Rosenbloom, January 21, 1960

This note is concerned with orthogonal polynomials on the unit circle and their use in probability theory.

Let $f(t) \geqq 0$ (not zero a.e.) be integrable on $-\pi \leqq t \leqq \pi$; then, according to Szegö [1], a system of polynomials $\left\{\phi_{n}(z)\right\}$ orthogonal with respect to $f(t)$ on $-\pi \leqq t \leqq \pi$ are uniquely determined by

(i) $\phi_{n}(z)$ is a polynomial of degree $n$ in which the coefficient of $z^{n}$ is real and positive,

(ii) $(1 / 2 \pi) \int_{-\pi}^{\pi} \phi_{n}(z) \phi_{m}(z) f(t) d t=\delta_{n m},\left(z=e^{i t}\right)$.

Recent results $[2 ; 3 ; 4]$ have shown the importance of the Szegö polynomials in discussing fluctuations of sums $S_{n}=X_{1}+\cdots$ $+X_{n},(n=0,1, \cdots)$, of independent, identically distributed random variables $X_{j}$. The results derived directly from the theory of the polynomials (1) were necessarily restricted to the case of symmetric, integral-valued random variables. We consider here an alternative definition of the polynomials (1) designed to allow a natural generalization of these results to nonsymmetric, not necessarily discretevalued random variables. This approach also seems to have connections with prediction theory.

Let $\left\{\alpha_{n}\right\}$ and $\left\{\beta_{n}\right\}$ be given sequences of complex numbers with $\alpha_{n} \beta_{n} \neq 1$ for all $n$, and let $u_{0}$ and $v_{0}$ be given constants. Then, the system

$$
\begin{aligned}
& u_{n}(z)-u_{n-1}(z)=\alpha_{n} z^{n} v_{n}(z), \\
& v_{n}(z)-v_{n-1}(z)=\beta_{n} z^{-n} u_{n}(z)
\end{aligned}
$$

determines polynomials $u_{n}(z)$ and $v_{n}(z)$ of at most degree $n$ in $z$ and $1 / z$, respectively. The condition $\alpha_{n} \beta_{n} \neq 1$ for all $n$ is necessary and sufficient for the existence of $u_{n}(z)$ and $v_{n}(z)$ for all $n$. Let $k_{n}^{2}$ $=\prod_{m=1}^{n}\left(1-\alpha_{m} \beta_{m}\right)^{-1}$, and set

$$
\phi_{n}(z)=z^{n} v_{n}(z) / k_{n}, \quad \psi_{n}(z)=z^{-n} u_{n}(z) / k_{n},
$$

where $k_{n}$ is one of the square roots of $k_{n}^{2}$ (we allow some arbitrariness here). We will connect $\phi_{n}(z)$ and $\psi_{n}(z)$ with the Szegö polynomials.

The following notation will be used consistently below. Let $f(t)$ be integrable on $-\pi \leqq t \leqq \pi$ with Fourier coefficients

1 This research was supported by the U. S. Air Force. 


$$
A_{k}=\frac{1}{2 \pi} \int_{-\pi}^{\pi} f(t) e^{-i k t} d t
$$

Let $D_{n}=\operatorname{det}\left(A_{j-i}\right),(i, j=0,1, \cdots, n)$, and let $E_{n}$ and $F_{n}$ denote the cofactors of $A_{-n}$ and $A_{n}$, respectively, in $D_{n}$.

Implicit in definition (1) is the following generalization. Let $f(t)$ be integrable on $-\pi \leqq t \leqq \pi$, and let $D_{n} \neq 0$ for all $n \geqq 0$; then, the systems of polynomials $\left\{\phi_{n}(z)\right\}$ and $\left\{\psi_{n}(z)\right\}$ are uniquely determined (to within a plus or minus sign) by

(i') $\phi_{n}(z)$ and $\psi_{n}(z)$ are polynomials of degree $n$ in $z$ and $1 / z$, respectively, with equal leading coefficients,

(ii') $(1 / 2 \pi) \int_{-\pi}^{\pi} \phi_{n}(z) \psi_{m}(z) f(t) d t=\delta_{n m},\left(z=e^{i t}\right)$.

The condition $D_{n} \neq 0$ for all $n$ is necessary and sufficient for the existence of $\phi_{n}(z)$ and $\psi_{n}(z)$ for all $n$.

Lemma 1. Let $f(t)$ be integrable on $-\pi \leqq t \leqq \pi$, and let $D_{n} \neq 0$ for all $n \geqq 0$. Then, the sequences $\alpha_{n}=E_{n} / D_{n-1}$ and $\beta_{n}=F_{n} / D_{n-1}$ with $u_{0}=v_{0}$ $=1 / A_{0}^{1 / 2}$ generate through (2) and (3) the same polynomials $\phi_{n}(z)$ and $\psi_{n}(z)$ determined by (4). Moreover, $k_{n}^{2}=A_{0} D_{n-1} / D_{n}$.

Lemma 1 shows the construction of $\alpha_{n}$ and $\beta_{n}$ given $f(t)$. How is $f(t)$ constructed given $\left\{\alpha_{n}\right\}$ and $\left\{\beta_{n}\right\}$ ? It can be shown using Lemma 1 that the $A_{k}$ 's $(k \geqq 1)$ are unique if an $f(t)$ exists ( $A_{0}$ arbitrary), and we are led to the moment problem. We consider here only the case $\sum\left|\alpha_{n}\right|<\infty$ and $\sum\left|\beta_{n}\right|<\infty$. In this case there exist unique functions $\phi^{+}(z)$ analytic in $|z|<1$ and $\phi^{-(z)}$ analytic in $|z|>1$ such that $\lim u_{n}(z)=\phi^{+}(z)$ uniformly in $|z| \leqq 1$ and $\lim v_{n}(z)=\phi^{-}(z)$ uniformly in $|z| \geqq 1$. For a special construction of $f(t)$ in terms of $\phi^{+}(z)$ and $\phi^{-}(z)$ see (b3) and Theorem 1.

The following theorems show in part an equivalence between two important classes of polynomial systems defined separately by (3) and (4). These two classes are

(a) the polynomials determined by (4) in case

$$
\begin{aligned}
f(t) & =\sum_{j=-\infty}^{\infty} A_{j} e^{i j t} \quad \text { with } \sum\left|A_{j}\right|<\infty, \\
D_{n} & \neq 0 \text { for all } n \geqq 0, \\
\log f(t) & =\sum_{j=-\infty}^{\infty} B_{j} e^{i j t} \quad \text { with } \sum\left|B_{j}\right|<\infty,
\end{aligned}
$$

(b) the polynomials determined by (3) in case

$$
\sum\left|\alpha_{n}\right|<\infty \text { and } \sum\left|\beta_{n}\right|<\infty, \quad u_{0}=v_{0},
$$




$$
\begin{aligned}
\alpha_{n} \beta_{n} & \neq 1 \text { for all } n \geqq 1, \\
\phi^{+}(z) & \neq 0 \text { in }|z| \leqq 1 \text { and } \phi^{-}(z) \neq 0 \text { in }|z| \geqq 1 .
\end{aligned}
$$

THEOREM 1. Let $\left\{\alpha_{n}\right\},\left\{\beta_{n}\right\}, u_{0}=v_{0}$ satisfy conditions (b1)-(b3). Then, $k_{\infty}^{2}=\lim k_{n}^{2}$ exists, and $\phi_{n}(z)$ and $\psi_{n}(z)$ from (3) satisfy (4) with $f(t)=k_{\infty}^{2} / \phi^{+}\left(e^{i t}\right) \phi^{-}\left(e^{i t}\right)$. Moreover, $f(t)$ satisfies (a1)-(a3).

Theorem 2. Let $f(t)=\sum_{j=-k}^{m} A_{j} e^{i j t}$ satisfy conditions (a1)-(a3). Then, $\alpha_{n}=E_{n} / D_{n-1}$ and $\beta_{n}=F_{n} / D_{n-1}$ (see Lemma 1) satisfy (b1) and (b2), and $\phi^{+}(z)$ and $\phi^{-}(z)$ satisfy (b3).

TheOREM 3. Let $f(t)$ satisfy conditions (a1)-(a3), and let $\alpha_{n}$ $=E_{n} / D_{n-1}$ and $\beta_{n}=F_{n} / D_{n-1}$ satisfy (b1). Then, $\alpha_{n}$ and $\beta_{n}$ satisfy (b2), and $\phi^{+}(z)$ and $\phi^{-}(z)$ satisfy (b3).

Theorem 2 could be proved for general $f(t)$ satisfying (a1)-(a3) if the following conjecture were proved.

Conjecture. Let $f(t)$ satisfy (a1) and (a2). Then, $f(t)$ satisfies (a3) if and only if $\sum\left|E_{n} / D_{n}\right|<\infty$ and $\sum\left|F_{n} / D_{n}\right|<\infty$. In either case

$$
\lim _{n \rightarrow \infty} D_{n} / D_{n-1}=\exp \left\{\frac{1}{2 \pi} \int_{-\pi}^{\pi} \log f(t) d t\right\} .
$$

Constructing orthogonal polynomials by system (2) leads naturally to a method of constructing orthogonal families in the continuous case. These orthogonal families are quite useful in solving fluctuation problems of sums $S_{n}$ in the nondiscrete case. The simplest continuous analogue of (2) is

$$
\begin{aligned}
& \frac{d}{d x} u(x, t)=\alpha(x) e^{i x t} v(x, t) \\
& u(0, t)=v(0, t)=1, \\
& \frac{d}{d x} v(x, t)=\beta(x) e^{-i x t} u(x, t)
\end{aligned}
$$

in which $\alpha(x)$ and $\beta(x)$ are given continuous functions of $x$ on $0 \leqq x<\infty$. Since $u(x, t)-1$ and $v(x, t)-1$ are Fourier transforms of functions which vanish outside the intervals $[0, x]$ and $[-x, 0]$, respectively, $u(x, z)$ and $v(x, z)$ are well-defined for all complex $z$. If $\alpha(x)$ and $\beta(x)$ are integrable on $[0, \infty)$, then there are unique functions $\phi^{+}(z)$ and $\phi^{-}(z)$ analytic in the upper and lower half-planes, respectively, such that $\lim u(x, z)=\phi^{+}(z),(x \rightarrow \infty)$, uniformly in $\operatorname{Im} z \geqq 0$ and $\lim v(x, z)=\phi^{-}(z),(x \rightarrow \infty)$, uniformly in $\operatorname{Im} z \leqq 0$. Let 


$$
\Phi(x, t)=\int_{0}^{x} e^{i y t} v(y, t) d y, \quad \Psi(x, t)=\int_{0}^{x} e^{-i y t} u(y, t) d y .
$$

TheOREM 4. Let $\alpha(x)$ and $\beta(x)$ be continuous and integrable on $0 \leqq x<\infty$, and let $\phi^{+}(z) \neq 0$ for $\operatorname{Im} z \geqq 0$ and $\phi^{-}(z) \neq 0$ for $\operatorname{Im} z \leqq 0$. Then,

$$
\lim _{A \rightarrow \infty} \frac{1}{2 \pi} \int_{-A}^{A} \Psi\left(x_{1}, t\right) \Phi\left(x_{2}, t\right) f(t) d t=\min \left(x_{1}, x_{2}\right),
$$

for all $x_{1}, x_{2} \geqq 0$, where $f(t)=1 / \phi^{+}(t) \phi^{-}(t)$.

The results stated here for the discrete case form the basis of a recent technical report by the author. Included in this report is an application to a fluctuation problem of a type first considered by Spitzer and Stone [3].

\section{REFERENCES}

1. U. Grenander and G. Szegö, Toeplitz forms and their applications, California Press, 1958.

2. M. Kac, Toeplitz matrices, translation kernels and a related problem in probability theory, Duke Math. J. vol. 21 (1954) pp. 501-509.

3. M. Kac, W. L. Murdock and G. Szegö, On the eigen-values of certain Hermitian forms, J. Rational Mech. Anal. vol. 2 (1935) pp. 767-800.

4. F. Spitzer and C. Stone, $A$ class of Toeplitz forms and their application to probability theory, to appear in Illinois J. Math.

University OF MinNesota 